\title{
A Weighted Splitting Approach For Low-Mach Number Flows
}

\author{
David Iampietro ${ }^{1,3}$, Frédéric Daude ${ }^{1}$, Pascal Galon ${ }^{4}$, and Jean-Marc Hérard ${ }^{2,3}$
}

\begin{abstract}
In steady-state regimes, water circulating in the nuclear power plants pipes behaves as a low Mach number flow. However, when steep phenomena occur, strong shock waves are produced. Herein, a fractional step approach allowing to decouple the convective from the acoustic effects is proposed. The originality is that the splitting between these two parts of the physics evolves dynamically in time according to the Mach number. The first one-dimensional explicit and implicit numerical results on a wide panel of Mach numbers show that this approach is as accurate and CPU-consuming as a state of the art Lagrange-Projection-type method.
\end{abstract}

\section{Introduction}

Even if it is intrinsically quasi-incompressible, water flowing inside nuclear plants can generate strong shock waves through which pressure can vary by dozens of bar. From a numerical point of view this diversity of behaviors raises a dilemma. Indeed, an efficient way to capture shocks in a fluid is to use exact or approximate Riemann solvers. However, stationary cases shown in [9] and theory developped in [7] prove that these latter are unable to maintain the approximated solution in the initial lowMach phase space also called "well-prepared space". What is more, they suffer from a serious loss of accuracy in case of low-Mach number flows. Eventually, the CFL condition inherent to explicit schemes requires very demanding non-dimensional timesteps bounded by the Mach number. One way to bypass these difficulties is to decouple convection from acoustic waves production by splitting the original conservation laws into two subsystems. Then, they can be successively solved and a

\footnotetext{
${ }^{1}$ EDF lab Saclay, 7 boulevard Gaspard Monge 92120 Palaiseau, e-mail: david.iampietro@edf.fr, e-mail: frederic.daude@edf.fr .

${ }^{2}$ EDF lab Chatou, 6 Quai Watier 78400 Chatou, e-mail: jean-marc.herard@edf.fr .

${ }^{3}$ I2M, UMR CNRS 7373 Technople Château-Gombert 39, rue F. Joliot Curie 13453 Marseille .

${ }^{4}$ CEA DEN/DANS/DM2S/SEMT/DYN, 91190 Saclay, e-mail: pascal.galon@ cea.fr .
} 
specific low-Mach number treatment or a direct implicitation can be done on the acoustic subsystem. Such a strategy has been tested in [4] where a transport and an acoustic subsystems are exhibited, the latter being reformulated in Lagrange variables. Inspired by the pioneering work of [1], the present approach introduces a splitting weighted by a parameter related to the instantaneous flow Mach number. By doing so, it becomes sensitive to any change of Mach regime, allows to capture shocks and may be accurate in the case of low-Mach number flows.

\section{A Weighted Splitting Approach}

Our work focuses on the compressible Euler system whom differential structure is similar to these of two-phase homogeneous models. In one dimension, the mass, momentum and energy conservation laws read:

$$
\begin{aligned}
& \partial_{t} \rho+\partial_{x}(\rho u)=0, \\
& \partial_{t}(\rho u)+\partial_{x}\left(\rho u^{2}+p\right)=0, \\
& \partial_{t}(\rho e)+\partial_{x}((\rho e+p) u)=0 .
\end{aligned}
$$

Here, $e=u^{2} / 2+\varepsilon$ is the specific total energy made of the kinetic contribution plus the specific internal energy $\varepsilon$ related to pressure and density by the equation of state $\varepsilon=\varepsilon^{E O S}(\rho, p)$. Eventually, one can introduce $c$ the sound speed such that $(\rho c)^{2}=\left(\partial_{p} \varepsilon_{\mid \rho}\right)^{-1}\left(p-\rho^{2} \partial_{\rho} \varepsilon_{\mid p}\right)$ which strongly depends on the fluid equation of state and governs the acoustic waves speed. Following [1], let us introduce $\mathscr{C}$ (respectively $\mathscr{A}$ ) a convective (respectively an acoustic) subsystem, namely:

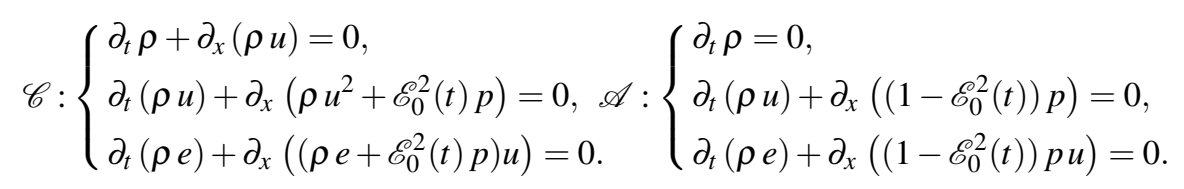

Here, $\mathscr{E}_{0}($.$\left.\left.) is a time-dependent weighting factor belonging to interval \right] 0,1\right]$. It is directly related to the maximal Mach number of the flow by the expression below:

$$
\mathscr{E}_{0}(t)=\min \left(M_{\max }(t), 1\right) ; \text { with: } M_{\max }(t)=\sup _{x \in \Omega}\left(M(x, t)=\frac{|u(x, t)|}{c(x, t)}\right),
$$

$\Omega$ being the computational domain. One can notice that formally summing conservative subsystems $\mathscr{C}$ and $\mathscr{A}$ allows to recover the original Euler system (1). In the case of a globally low-Mach number flow, $M_{\max }(t) \approx \mathscr{E}_{0}(t) \ll 1$, and pressure terms completely disappear from $\mathscr{C}$ which turns out to be a pure "convective" subsystem. Pressure terms are treated afterwards in $\mathscr{A}$ which becomes an "acoustic" subsystem. Actually, a low-Mach correction or a straight time-implicit resolution applied on its flux would allow to reduce the numerical diffusion or remove the most constraining part of the CFL condition. However, suppose that at instant $t$ the flow is 
such that $M_{\max }(t)$ jumps to 1 suddenly. Then, $\mathscr{E}_{0}(t)$ will be close to $1, \mathscr{C}$ formally converges towards the full Euler system while $\mathscr{A}$ is a degenerated stationary subsystem. Hence, if $\mathscr{C}$ is solved using a time-explicit Godunov-like scheme, Euler shocks would be optimally captured. This strategy relies on the hypothesis that the waves produced by $\mathscr{C}$ and $\mathscr{A}$ are real and also that their asymptotic behavior in terms of $\mathscr{E}_{0}$ is the expected one. The proposition below clarifies this point (see [10]):

\section{Proposition 1 (Hyperbolicity of convective and acoustic subsystems).}

Let us introduce $c_{\mathscr{C}}(\rho, p)$ and $c_{\mathscr{A}}(\rho, p)$ two modified sound speeds such that:

$$
\begin{aligned}
& \left(\rho c_{\mathscr{C}}(\rho, p)\right)^{2}=\left(\partial_{p} \varepsilon_{\mid \rho}\right)^{-1}\left(\mathscr{E}_{0}^{2} p-\rho^{2} \partial_{\rho} \varepsilon_{\mid p}\right), \\
& \left(\rho c_{\mathscr{A}}(\rho, p)\right)^{2}=\left(\partial_{p} \varepsilon_{\mid \rho}\right)^{-1} p .
\end{aligned}
$$

In case of a stiffened gas thermodynamics, $c_{\mathscr{C}}^{2} \geq 0$. Besides, if pressure remains positive, $c_{\mathscr{A}}^{2} \geq 0$. Under this condition, the subsystems $\mathscr{C}$ and $\mathscr{A}$ are hyperbolic. The eigenvalues of $\mathscr{C}$ and $\mathscr{A}$ are:

$$
\begin{array}{ll}
\lambda_{1}^{\mathscr{C}}=u-\mathscr{E}_{0} c_{\mathscr{C}} & \leq \lambda_{2}^{\mathscr{C}}=u \leq \lambda_{3}^{\mathscr{C}}=u+\mathscr{E}_{0} c_{\mathscr{C}}, \\
\lambda_{1}^{\mathscr{A}}=-\left(1-\mathscr{E}_{0}^{2}\right) c_{\mathscr{A}} & \leq \lambda_{2}^{\mathscr{A}}=0 \leq \lambda_{3}^{\mathscr{A}}=\left(1-\mathscr{E}_{0}^{2}\right) c_{\mathscr{A}},
\end{array}
$$

the 1-wave and 3-wave of both subsystems are associated to genuinely non-linear fields whereas the 2-wave field are linearly degenerate.

\section{Suliciu-like Relaxation Schemes To Solve $\mathscr{C}$ and $\mathscr{A}$}

Relaxation schemes emerge from the theory of kinetic schemes described in [2, 3]. As shown in [5], such a method can be applied on a rather general fluid model endowed with a set of conservation laws, a strictly convex entropy and basic thermodynamical constraints linking state variables. Following the Suliciu-like relaxation method, also used in [4], let us introduce $\mathscr{C}^{\mu}$ (respectively $\mathscr{A}^{\mu}$ ) the relaxed convective (respectively the relaxed acoustic) subsystem:

$$
\begin{gathered}
\mathscr{C}^{\mu}:\left\{\begin{array}{l}
\partial_{t} \rho+\partial_{x}(\rho u)=0, \\
\partial_{t}(\rho u)+\partial_{x}\left(\rho u^{2}\right)+\partial_{x}\left(\mathscr{E}_{0}^{2}(t) \Pi\right)=0, \\
\partial_{t}(\rho e)+\partial_{x}\left(\left(\rho e+\mathscr{E}_{0}^{2}(t) \Pi\right) u\right)=0, \\
\partial_{t}(\rho \Pi)+\partial_{x}\left(\left(\rho \Pi+a_{\mathscr{C}}^{2}\right) u\right)=\frac{\rho}{\mu}(p-\Pi),
\end{array}\right. \\
\mathscr{A}^{\mu}:\left\{\begin{array}{l}
\partial_{t} \rho=0, \\
\partial_{t}(\rho u)+\partial_{x}\left(\left(1-\mathscr{E}_{0}^{2}(t)\right) \Pi\right)=0, \\
\partial_{t}(\rho e)+\partial_{x}\left(\left(1-\mathscr{E}_{0}^{2}(t)\right) \Pi u\right)=0, \\
\partial_{t}(\rho \Pi)+\partial_{x}\left(\left(1-\mathscr{E}_{0}^{2}(t)\right) a_{\mathscr{A}}^{2} u\right)=\frac{\rho}{\mu}(p-\Pi) .
\end{array}\right.
\end{gathered}
$$


Here, $\frac{\rho}{\mu}(p-\Pi)$ can be formally interpreted as a correction term of time scale $\mu$ forcing the relaxed pressure $\Pi$ to converge towards the physical pressure instantaneously if $\mu$ tends to zero. Besides, $a_{\mathscr{C}}$ and $a_{\mathscr{A}}$ are the constant relaxation coefficients encapsulating the thermodynamical nonlinearity. What is more, under the subcaracteristic condition $a_{\mathscr{C}}>\rho c_{\mathscr{C}}$ (respectively $a_{\mathscr{A}}>\rho c_{\mathscr{A}}$ ), $\mathscr{C}^{\mu}$ (respectively $\mathscr{A}^{\mu}$ ) converges formally towards $\mathscr{C}$ (respectively $\mathscr{A}$ ) at order one in $\mu$. Then, the augmented set of conservation laws is still hyperbolic and all its fields are linearly degenerate. Hence, it is possible to derive an exact Godunov solver for these relaxed subsystems. The eigenvalues of $\mathscr{C}^{\mu}$ are $u-\mathscr{E}_{0} a_{\mathscr{C}} \tau, u$ and $u+\mathscr{E}_{0} a_{\mathscr{C}} \tau$ with $\tau=1 / \rho$ the specific volume. The ones of $\mathscr{A}^{\mu}$ are $-\left(1-\mathscr{E}_{0}^{2}\right) a_{\mathscr{A}} \tau, 0$, and $\left(1-\mathscr{E}_{0}^{2}\right) a_{\mathscr{A}} \tau$. The numerical flux related to $\mathscr{C}$ (respectively $\mathscr{A}$ ) is derived by solving a convective (respectively an acoustic) Riemann problem associated to $\mathscr{C}^{\mu}$ (respectively $\mathscr{A}^{\mu}$ ). In the end, for an explicit time integration, the convective flux at face $i+1 / 2$ and time $t^{n}$ reads:

$$
\mathbf{H}_{\mathbf{c}_{i+1 / 2}}^{n}=\left\{\begin{array}{l}
\frac{1}{2}\left(\mathbf{F}_{\mathscr{C}}\left(\mathbf{U}_{i}^{n}\right)+\mathbf{F}_{\mathscr{C}}\left(\mathbf{U}_{i+1}^{n}\right)\right) \\
-\frac{1}{2}\left|u_{i}^{n}-\mathscr{E}_{0}^{n}\left(a_{\mathscr{C}}^{n}\right)_{i+1 / 2} \tau_{i}^{n}\right|\left(\mathbf{U}_{i+1 / 2}^{*, n}-\mathbf{U}_{i}^{n}\right) \\
-\frac{1}{2}\left|\left(u_{\mathscr{C}}^{*}\right)_{i+1 / 2}^{n}\right|\left(\mathbf{U}_{i+1 / 2}^{* *, n}-\mathbf{U}_{i+1 / 2}^{*, n}\right) \\
-\frac{1}{2}\left|u_{i+1}^{n}+\mathscr{E}_{0}^{n}\left(a_{\mathscr{C}}^{n}\right)_{i+1 / 2} \tau_{i+1}^{n}\right|\left(\mathbf{U}_{i+1}^{n}-\mathbf{U}_{i+1 / 2}^{* *, n}\right),
\end{array}\right.
$$

with $\mathbf{F}_{\mathscr{C}}(\underline{U})=\left[\rho u, \rho u^{2}+\mathscr{E}_{0}^{2} p,\left(\rho e+\mathscr{E}_{0}^{2} p\right) u\right]^{T}$, and:

$$
\begin{aligned}
& \mathbf{U}_{i+1 / 2}^{*, n}=\left[\begin{array}{c}
\left(\rho_{i, \mathscr{C}}^{*}\right)^{n} \\
\left(\rho_{i, \mathscr{C}}^{*}\right)^{n}\left(u_{\mathscr{C}}^{*}\right)_{i+1 / 2}^{n} \\
\left(\rho_{i, \mathscr{C}}^{*}\right)^{n}\left(e_{i, \mathscr{C}}^{*}\right)^{n}
\end{array}\right], \mathbf{U}_{i+1 / 2}^{* *, n}=\left[\begin{array}{c}
\left(\rho_{i+1, \mathscr{C}}^{*}\right)^{n} \\
\left(\rho_{i+1, \mathscr{C}}^{*}\right)^{n}\left(u_{\mathscr{C}}^{*}\right)_{i+1 / 2}^{n} \\
\left(\rho_{i+1, \mathscr{C}}^{*}\right)^{n}\left(e_{i+1, \mathscr{C}}^{*}\right)^{n}
\end{array}\right], \\
& \left(a_{\mathscr{C}}^{n}\right)_{i+1 / 2}=\operatorname{Kmax}\left(\rho_{i}^{n}\left(c_{\mathscr{C}}\right)_{i}^{n}, \rho_{i+1}^{n}\left(c_{\mathscr{C}}\right)_{i+1}^{n}\right), K>1 .
\end{aligned}
$$

The expressions of intermediate quantities like $\left(u_{\mathscr{C}}^{*}\right)_{i+1 / 2}^{n},\left(\rho_{k, \mathscr{C}}^{*}\right)^{n}$ and $\left(e_{k, \mathscr{C}}^{*}\right)^{n}, k \in$ $\{i, i+1\}$ are close to these derived in [6, 4]. More details are given in [10]. The acoustic flux is simpler because of the zero eigenvalue:

$$
\begin{gathered}
\text { with: }\left\{\begin{array}{l}
\left(u_{\mathscr{A}}^{*}\right)_{i+1 / 2}^{n}=\frac{u_{i+1}^{n}+u_{i+1 / 2}^{n}}{2}=\left(1-\left(\mathscr{E}_{0}^{n}\right)^{2}\right)\left[\begin{array}{c}
0 \\
\left(\Pi_{\mathscr{A}}^{*}\right)_{i+1 / 2}^{n, \theta} \\
\left(\Pi_{\mathscr{A}}^{*}\right)_{i+1 / 2}^{n, \theta}\left(u_{\mathscr{A}}^{*}\right)_{i+1 / 2}^{n}
\end{array}\right], \\
\left(\Pi_{\mathscr{A}}^{*}\right)_{i+1 / 2}^{n, \theta}=\frac{1}{2\left(a_{\mathscr{A}}\right)_{i+1 / 2}^{n}}\left(p_{i+1}^{n}-p_{i}^{n}\right), \\
\left(a_{\mathscr{A}}^{n}\right)_{i+1 / 2}^{n}=K \max \left(\rho_{i}^{n}\left(c_{\mathscr{A}}\right)_{i}^{n}, \rho_{i+1}^{n}\left(c_{\mathscr{A}}\right)_{i+1}^{n}\right), K>1 .
\end{array}\right.
\end{gathered}
$$


Following [7], the parameter $\theta_{i+1 / 2}^{n}$ introduced in (7) is a low-Mach number correction term. Indeed, $\theta_{i+1 / 2}^{n}=1$ is the original formula with no correction, $\theta_{i+1 / 2}^{n}=\left|\left(u_{\mathscr{A}}^{*}\right)_{i+1 / 2}^{n}\right| / \max \left(c_{i}^{n}, c_{i+1}^{n}\right)$ prevents an initial well-prepared solution from leaving the well-prepared space after one iteration. It also diminishes the numerical diffusion in the low-Mach number configurations. See $[9,7,4]$ for more details. The discrete expression of the weighting parameter $\mathscr{E}_{0}$ follows the continuous definition written in (2):

$$
\mathscr{E}_{0}^{n}=\max \left(\mathscr{E}_{\text {inf }}, \min \left(M_{\max }^{n}, 1\right)\right) ; \text { with: } M_{\max }^{n}=\max _{i \in\left[1, N_{\text {cells }}\right]}\left(\frac{\left|u_{i}^{n}\right|}{c_{i}^{n}}\right) .
$$

Here, $0<\mathscr{E}_{\text {inf }} \ll 1$ is only a lower bound preventing $\mathscr{E}_{0}^{n}$ from being exactly equal to zero if velocity is initially null everywhere. Finally, the overall dynamical fractional step approach can be summed up in the following equations:

$$
\begin{aligned}
& \mathscr{C}:\left\{\begin{array}{l}
\mathbf{U}_{i}^{n+}=\mathbf{U}_{i}^{n}-\frac{\Delta t}{\Delta x}\left(\mathbf{H}_{\mathbf{c}_{i+1 / 2}}\left(\mathbf{U}_{i}^{n}, \mathbf{U}_{i+1}^{n}\right)-\mathbf{H}_{\mathbf{c}_{i-1 / 2}}\left(\mathbf{U}_{i-1}^{n}, \mathbf{U}_{i}^{n}\right)\right), \\
\Pi_{i}^{n+}=p^{E O S}\left(\mathbf{U}_{i}^{n+}\right)=p_{i}^{n+}
\end{array}\right. \\
& \mathscr{A}:\left\{\begin{array}{l}
\mathbf{U}_{i}^{n+1}=\mathbf{U}_{i}^{n+}-\frac{\Delta t}{\Delta x}\left(\mathbf{H}_{\mathbf{a c}_{i+1 / 2}}\left(\mathbf{U}_{i}^{n+}, \mathbf{U}_{i+1}^{n+}\right)-\mathbf{H}_{\mathbf{a c}_{i-1 / 2}}\left(\mathbf{U}_{i-1}^{n+}, \mathbf{U}_{i}^{n+}\right)\right), \\
\Pi_{i}^{n+1}=p^{E O S}\left(\mathbf{U}_{i}^{n+1}\right)=p_{i}^{n+1}
\end{array}\right.
\end{aligned}
$$

We also have the following results (see [10] for a proof):

\section{Proposition 2 (Conservativity, Positivity, Low-Mach Accuracy).}

- Conservativity: The overall scheme (9) is conservative.

- Positivity: Assume $\forall i: \rho_{i}^{n}>0, \varepsilon_{i}^{n}>0$. Then, $\rho_{i}^{n+1}>0, \varepsilon_{i}^{n+1}>0$ is ensured under modified subcaracteristic conditions:

$$
\begin{aligned}
& \left(a_{\mathscr{C}}^{n}\right)_{i+1 / 2}=K \max \left(\rho_{i}^{n}\left(c_{\mathscr{C}}\right)_{i}^{n}, \rho_{i+1}^{n}\left(c_{\mathscr{C}}\right)_{i+1}^{n}, a_{i}^{\rho ; \varepsilon, n}, a_{i+1}^{\rho ; \varepsilon, n}\right) \\
& \left(a_{\mathscr{A}}^{n}\right)_{i+1 / 2}=K \max \left(\rho_{i}^{n}\left(c_{\mathscr{A}}\right)_{i}^{n}, \rho_{i+1}^{n}\left(c_{\mathscr{A}}\right)_{i+1}^{n}, a_{i}^{\rho ; \varepsilon, n}, a_{i+1}^{\rho ; \varepsilon, n}\right)
\end{aligned}
$$

where the non-dimensional expressions associated with $a_{i}^{\rho ; \varepsilon, n}$ and $a_{i+1}^{\rho ; \varepsilon, n}$ are of order $O(1)$; and under a global CFL condition: $\Delta t^{n}=\min \left(\Delta t_{E}^{n}, \Delta t_{\mathscr{C}}^{n}, \Delta t_{\mathscr{A}}^{n}\right)$, with $\Delta t_{E}^{n}$ (respectively $\Delta t_{\mathscr{C}}^{n}, \Delta t_{\mathscr{A}}^{n}$ ) the timestep bounded by the Euler (respectively $\mathscr{C}, \mathscr{A}) C F L$ condition garanteeing no interaction between waves produced by the face Riemann problems.

- Low-Mach accuracy: Assume that the initial conditions belong to the wellprepared space (see [7] for a definition) and that $\mathscr{E}_{0}$ is given by (8). Then, the non-dimensional numerical diffusion of a smooth solution computed thanks to the scheme is a $O(\Delta x)$ instead of $O(\Delta x / M)$ if the above global CFL condition holds, and if the discrete low-Mach correction $\theta_{i+1 / 2}^{n}$ is triggered. 


\section{Numerical Results}

We perform a one-dimensional Sod-type shock tube. The fluid is endowed of an ideal gas thermodynamics with $\gamma=7 / 5$. The initial data are: $\rho_{L}^{0}=1 \mathrm{~kg} \cdot \mathrm{m}^{-3}$, $u_{L}^{0}=0 \mathrm{~m} . \mathrm{s}^{-1}, p_{0, L}=p_{0, R}(1+\varepsilon), \rho_{R}^{0}=0.125 \mathrm{~kg} . \mathrm{m}^{-3}, u_{R}^{0}=0 \mathrm{~m} . \mathrm{s}^{-1}, p_{0, R}=0.1 \mathrm{bar}$. By tuning $\varepsilon$, the maximal flow Mach number can be modified. Fig. 1 shows the pressure convergence curves for three different Mach values: $M=0.92, M=9.5 \times 10^{-2}$ and $M=4.2 \times 10^{-3}$. The cells number varies from $10^{2}$ to $9 \times 10^{4}$. Five different schemes have been tested: "no-Sp" corresponds to the case where $\mathscr{E}_{0} n=1$ is imposed along the simulation. Thus, the splitting is not triggered. "Sp- $(\sqrt{M})$ " is the weighted splitting approach with $\mathscr{E}_{0}^{n}=\max \left(\mathscr{E}_{\text {inf }}, \min \left(\sqrt{M_{\text {max }}^{n}}, 1\right)\right)$ while "Sp- $(M)$ " involves $\mathscr{E}_{0}$ defined in formula (8) which is optimal, because, as proven in [10], it minimizes the numerical diffusion of the subsystem $\mathscr{C}$ in the low-Mach number case. Eventually, "LP" is the Lagrange Projection splitting method, fully described in [4] and taken as a benchmark. "-corr" means that the low-Mach correction is triggered.

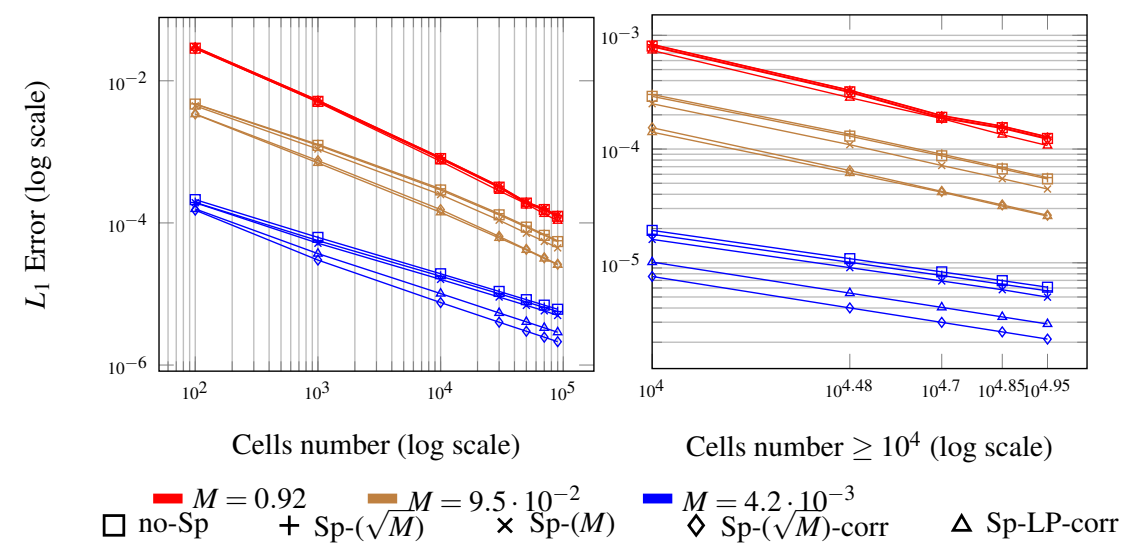

Fig. 1: Pressure Convergence Curves: Explicit Schemes

One can notice that, when $M \approx 1$, all the schemes are equivalent in terms of accuracy. In the sequel, as the Mach number decreases the low-Mach corrected schemes become the most accurate ones. Particularly, Sp- $(\sqrt{M})$-corr seems to be more precise than Sp-LP-corr at $M=4.2 \times 10^{-3}$. However, one should notice that for every schemes, the order of convergence is depreciated as the Mach number decreases. Indeed for pressure, it passes from 0.87 at $M=0.92$ (the expected order already obtained in [8]) to 0.82 at $M=9.5 \times 10^{-2}$ and 0.56 in the low-Mach case. Velocity profiles are plotted on Fig. 2. It seems that the accuracy of Sp- $(M)$ is higher than these of $\mathrm{Sp}-(\sqrt{M})$ through the left rarefaction wave where the exact solution is continuous. Besides, the low-Mach correction applied on the weighted splitting approach results in small overshoots located in the tail of the left rarefaction wave and before the shock front.

Instead of using the low-Mach correction, one can directly apply an implicit approximation of the acoustic flux (6) using a method that relies on strong re- 


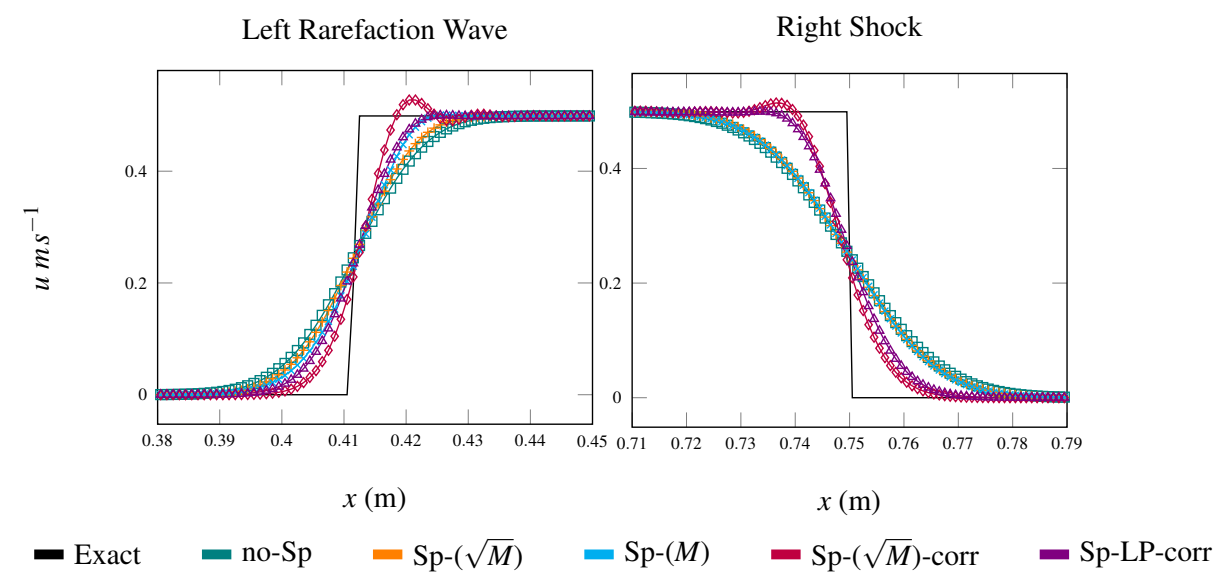

Fig. 2: Velocity Profiles: $M=4.2 \times 10^{-3}, N_{\text {cells }}=10^{3}$

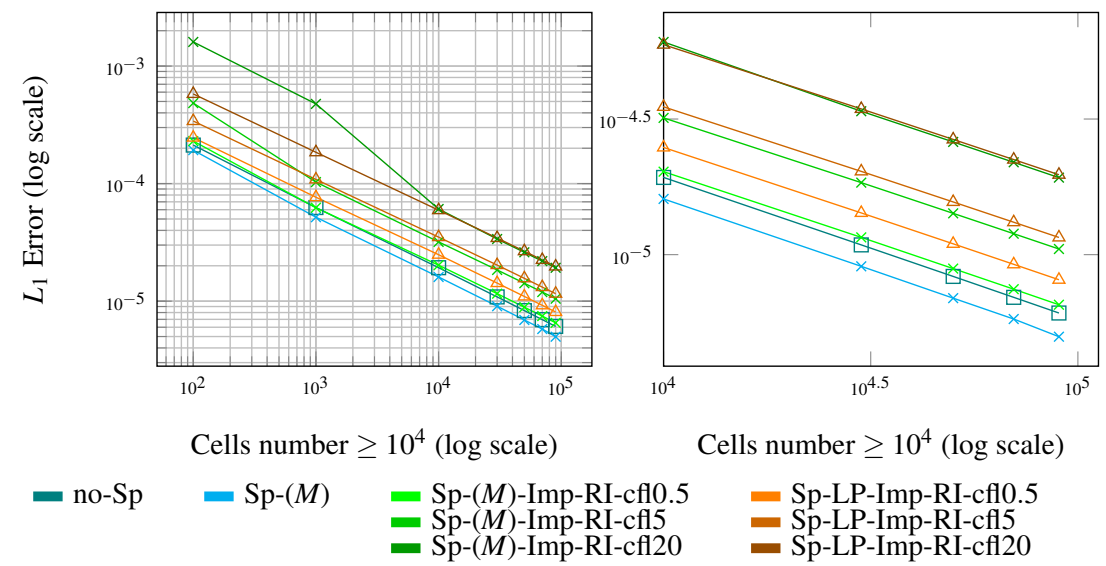

Fig. 3: Pressure Convergence Curves: Implicit Schemes, $M=4.2 \cdot 10^{-3}$

laxed Riemann Invariants (see [6] and [4] for more details). Pressure convergence curves for different implicit schemes at $M=4.2 \times 10^{-3}$ are shown on Fig. 3. Here, $\forall k \in\{1 / 2,5,20\}$, the mention "-cfl $[k]$ " indicates that the Courant number involved in the determination of $\Delta t_{E}^{n}$ and $\Delta t_{\mathscr{A}}^{n}$ defined in Proposition 2 is equal to " $k$ ". As expected, the implicit techniques are more diffusive than explicit schemes. Besides, at a given mesh, CPU time diminishes considerably as the Courant number increases. For example, at $N_{\text {cells }}=10^{3}, \mathrm{Sp}$ - $(M)$-Imp-RI-cfl0.5 takes $10.1 \mathrm{~s}$ whereas $\mathrm{Sp}-(M)$-Imp-RI-cfl5 (respectively Sp- $(M)$-Imp-RI-cfl20) requires $1.9 s$ (respectively $0.7 s)$. Finally, one can notice that the present implicit weighted splitting approach is as accurate as the implicit Lagrange-Projection method. In the low-Mach regime, the trade-off between explicit-accuracy versus the implicit-CPU-rapidity is solved thanks to the efficiency curve plotted on Fig. 4. At a given precision, for low-Mach 
unsteady cases, explicit schemes are still less CPU-consuming than implicit techniques.

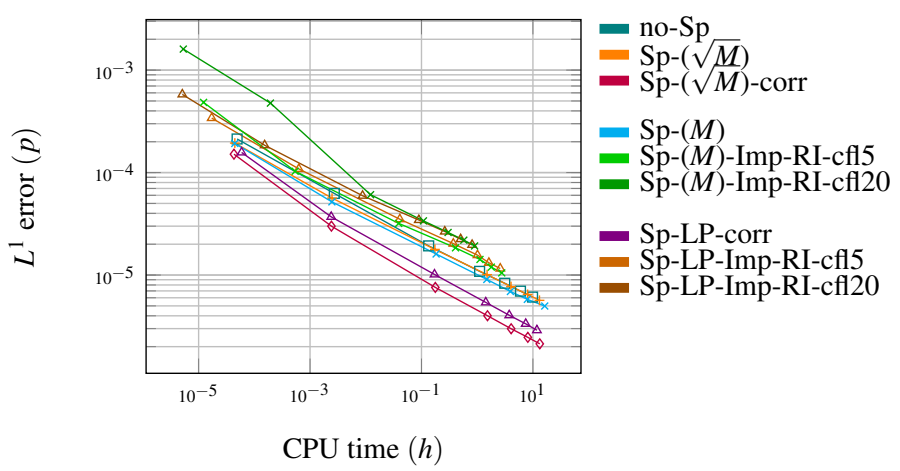

Fig. 4: Pressure Efficiency Curve: $M=4.2 \times 10^{-3}$

Acknowledgement: D. Iampietro received a financial support by ANRT through an EDF-CIFRE contract 2015/0561. Numerical facilities were provided by EDF.

\section{References}

1. Baraille, R., Bourdin, G., Dubois, F., Le Roux, A.Y.: Une version à pas fractionnaires du schéma de Godunov pour l'hydrodynamique. Compte Rendu de 1'Académie des Sciences 314, 147-152 (1992)

2. Bouchut, F.: Entropy satisfying flux vector splittings and kinetic BGK models. Numerische Mathematik 94, 623-672 (2003)

3. Bouchut, F.: Nonlinear Stability of Finite Volume Methods for Hyperbolic Conservation Laws. Birkäser (2004)

4. Chalons, C., Girardin, M., Kokh, S.: An all-regime Lagrange-Projection like scheme for the gas dynamics equations on unstructured meshes. Communications in Computational Physics 20, 188-233 (2016)

5. Coquel, F., Godlewski, E., Seguin, N.: Relaxation of fluid systems. Mathematical Models and Methods in Applied Science 22, 43-95 (2012)

6. Coquel, F., Nguyen, Q.L., Postel, M., Tran, Q.H.: Entropy-satisfying relaxation method with large time-steps for Euler IBVPS. Mathematics of Computation 79, 1493-1533 (2010)

7. Dellacherie, S., Omnes, P., Jung, J., Raviart, P.: Construction of modified Godunov type schemes accurate at any Mach number for the compressible Euler system. Mathematical Models and Methods in Applied Science 26, 2525-2615 (2016)

8. Gallouët, T., Hérard, J.M., Seguin, N.: Some recent finite volume schemes to compute Euler equations using real gas EOS. International Journal for Numerical Methods in Fluids 39, 1073-1138 (2002)

9. Guillard, H., Murrone, A.: On the behavior of upwind schemes in the low Mach number limit: II Godunov type schemes. Computers and Fluids 33, 655-675 (2004)

10. Iampietro, D., Daude, F., Galon, P., Hérard, J.M.: A Mach-sensitive splitting approach for Euler-like systems (2017). Https://hal.archives-ouvertes.fr/hal-01466827 